\title{
Progress on Therapy of Infertility by Integrated Traditional Chinese and Western Medicine
}

\author{
Shaohua Wang ${ }^{1}$, Jianli Jiang², Xiaobing Lu ${ }^{3}$ \\ ${ }^{1}$ The Second People's Hospital of Yichang, Yichang, China \\ ${ }^{2}$ The First People's Hospital of Yichang, Yichang, China \\ ${ }^{3}$ The Institute of Mechanics, Chinese Academy of Sciences, Beijing, China \\ Email: xblu@imech.ac.cn
}

How to cite this paper: Wang, S.H., Jiang, J.L. and Lu, X.B. (2020) Progress on Therapy of Infertility by Integrated Traditional Chinese and Western Medicine. Advances in Reproductive Sciences, 8, 175-185. https://doi.org/10.4236/arsci.2020.84015

Received: August 12, 2020

Accepted: September 12, 2020

Published: September 15, 2020

Copyright (c) 2020 by author(s) and Scientific Research Publishing Inc. This work is licensed under the Creative Commons Attribution International License (CC BY 4.0).

http://creativecommons.org/licenses/by/4.0/

\begin{abstract}
Infertility is one of the difficult complicated diseases. Many couples suffer from it. The pathogenesis is very complicated. The imbalance or lesson of any link of the reproductive system can cause infertility. This paper summarizes the treatment of female infertility by integrated Traditional Chinese Medicine (TCM) and Western Medicine (WM) which can not only improve the ovulation rate and pregnancy rate, but also decrease the complications. The effects are better than that by TCM or WM only. Therefore, the coupling method is worth to be used widely in clinical practice.
\end{abstract}

\section{Keywords}

Infertility, Treatment, Integrated Method, Traditional Chinese Medicine, Western Medicine

\section{Introduction}

The definition of infertility is that one couple has not a pregnancy after they live together for one year and have a normal sex life without contraception. If one couple never conceives after marriage, it is called primary infertility. If they have a pregnancy once but fail in pregnancy for one year without contraception, it is called secondary infertility. Morbidity of infertility is different with nation, ethnicity and living region. Man and woman can both cause infertility. Infertility due to woman is about $40 \%$ of the total. The factors causing infertility due to women include ovulation disorder and oviduct ovulation disorder etc. [1].

The use of west drugs for ovulation disorder can cause high ovulation rate and low pregnancy. Multiple pregnancy and ovarian hyperstimulation syndrome are also the complications. TCM has long term curative effects without obvious side 
effects, but the circle of treatment is long. If TCM combines the WM, not only the ovulation rate and pregnancy rate can rise, but also the complications can decrease. The coupling method is worth to be used widely in clinical practice.

For example, menstrual cycle therapy of TCM is combined with the artificial cycle therapy of WM to treat infertility. This method can choose corresponding treatment principle and method according to the period of menstrual circle, because menstrual cycle therapy is based on pathogenesis obtained through differentiation of symptoms and signs. The treatment induces the menstruation to become normal and the infertility is cured by adjusting the organs, meridians, blood and Ying/Yang.

The therapeutic methods of WM for anovulatory infertility include medication and surgical treatment. The medicine is used alone or in combination with each link of the hypothalamus pituitary ovary axis. Clinically, ovulation stimulation drugs are mainly clomiphene citrate, aromatase inhibitors, gonadotropin, gonadotropin releasing hormone etc. The clinical efficacy by ovulation stimulation drugs is fast, but the side effects such as ovarian hyperstimulation syndrome, premature luteinized unruptured follicle syndrome, multiple pregnancy are often observed. The surgical treatment includes ovarian drilling and model ovariectomy. The treatment of TCM is mainly to tonify the kidney based on the judgement of kidney deficiency, accompanying soothing liver and regulating qi. By this way to promote the blood circulation and remove the blood stasis etc., Cycle therapy of TCM based on the knowledge of regular changes of female menstrual cycle has been applied and extended. However, the long circle of treatment is the main problem of TCM. The best choice is the combination of TCM and the WM.

In this paper, the pathogenesis of infertility is first introduced. Then, the progress in treatment of infertility by coupling TCM and WM is summarized according to the types of infertility. For each type of infertility, the pathogeny and the treatment method are introduced carefully. At last, the trend of study and treatment of infertility in the future is discussed.

\section{Pathogenesis of Infertility}

The imbalance or lesson of any link of the reproductive system can cause infertility. The male infertility is mainly caused by semen abnormality and abnormal spermatogenesis. The factors of female infertility include ovulation disorder, abnormal fallopian tube, endometriosis, immunity etc.

Hyperprolactinemia, a kind of endocrine diseases that the level of serum prolactin is over $25 \mathrm{ng} / \mathrm{mL}$ ad $2 \mathrm{vic}$, is a common reason of infertility. The main features are amenorrhea, galactorrhea [2].

WM believes that high prolactin inhibits the function of hypothalamuspituitary-gonadal axis, thereby affecting hormone secretion and ovarian function, resulting in infertility. TCM considers that the disease is caused by the imbalance of "kidney-Tiangui-Chong and Ren-uterus". The dysfunctional of liver, 
spleen and kidney is the root cause. Therefore, WM is mainly treated with dopamine receptor agonist, but it has toxic side effects and high recurrence rate. TCM and acupuncture can comprehensively adjust the endocrine and reproductive function of human body. TCM can also use the idea of "treating the same disease with different therapies" to make individualized comprehensive treatment scheme [3]. The curative effect is remarkable, but the circle of treatment is long and the effect is slow [4].

\section{Main Female Infertilities}

\subsection{Infertility Due to Diminished Ovarian Reserve}

Ovarian reserve means the ability of follicular growth in cortical area of ovary, and developing into a fertilizable oocyte, including the number and the quality of follicles in ovary. Diminished ovarian reserve means that the number of intraovarian follicles decreases and the quality of oocyte reduces. The main manifestations are less menstruation, prolonged menstruation, accompanied soreness and weakness of the lumbar and knees region, insomnia and dreaminess, or further developed into premature ovarian failure and infertility. It is thought that the decrease of ovarian reserve is the result of the interaction of various factors. Normally, the ovarian reserve decreases after 30 years old. With the acceleration of the social rhythm and the rise of mental stress and environmental pollution, the incidence rate of ovarian reserve decrease increases gradually and tends to be younger. Decrease of ovarian reserve affects not only the normal pregnancy, but also the in vitro fertilization combined with embryo transfer.

In TCM for treating diminished ovarian reserve, most frequently used herb kind is tonify deficiency medicine. The liver and kidney are the most frequent channel tropisms. The warm-natured and sweet drugs are often used. The aim of the treatment is to tune up kidney-invigoration. The method of WM is to protect the remained ovarian function, build artificial circle by supplying the hormone and ovulation induction. Hormone replacement therapy is the main treatment method for this disease. The main drugs are estrogen and progesterone, clomiphene citrate [5]. TCM combined with WM can not only improve the ovarian function, but also avoid the adverse reactions caused by adopting only WM. The total effect is better than that by only TCM or WM.

\subsection{Immune Infertility}

Clinically, the immune infertility is thought as that there is evidence of anti-reproductive immunity, but there is not ovulation and reproductive dysfunction in women and no obvious abnormality in semen routine examination. It is reported that $10 \% \sim 20 \%$ of the infertility is due to immune factors [6]. The immune infertility includes anti-sperm antibody, endometrial antibody, anti-ovarian antibody, anti-HCG antibody, anti-zona pellucida antibody, anti-trophoblast antibody, anticardiolipin antibody, cell factor, blocking antibody etc. [7]. 
Based on the theory of deficiency of kidney-Qi and imbalance of Qi and blood in Chong and Ren for the immune infertility, TCM presents theories of toxic heat inside, damp-heat pouring downward, blockage of Qi and blood stasis. Liu et al. [8] thought that the immune infertility is caused by the deficiency of kidney-Qi and imbalance of Qi and blood which can lead to the blockage of Qi and blood stasis. The pregnancy is then disturbed. Yu [9] thought that the immune infertility is due to the deficiency of vital energy and so toxin invades the uterine vessels, blood stasis accumulates in the uterus, damp and heat blocks the channels. These factors disturb the Qi and blood, and makes the Qi in Chong and Ren disorder, the power accepting the sperms is then lost.

The treatment method of WM for the immune infertility is to adopt immunosuppressant. Glucocorticoid is the common drug for the immune infertility. It can reduce the occurrence of antibody and the conjugation of antibody and antigen by inhibition of cell factor and lymphoid growth factor. The other choice is to use anticoagulant drugs. For example, the use of immunosuppressant combined with anticoagulant is the recognized treatment plan for the patients with positive anticardiolipin antibody. Aspirin and low molecular weight heparin are the main anticoagulant drugs at present. For patients of positive anti-sperm antibody, the method of device contraception is often used. The use of device contraception accompanying the immunosuppressant can reduce the stimulation of sperm antigen to the genital tract. For patients of positive anti-ovarian antibody, ovarian function should be improved besides the use of immunosuppressant. For patients of positive anti-endometrial antibody, the status of endometriosis must be corrected.

The treatment methods of TCM on immune infertility include periodic therapy, syndrome-differentiation therapy, acupuncture therapy etc. [10]. The types according to the syndrome differentiation of TCM on immune infertility have dampness and heat stasis, Yang deficiency and blood stasis, fire excess from Yin deficiency, Qi deficiency and blood stasis. Therefore, the treatments are mainly tonifying kidney and replenishing qi, activating blood and dissolving stasis, nourishing Yin, heat clearing and dehumidification. These methods have obvious effects on the treatment of immune infertility.

\subsection{Infertility due to Ovulation Disturbance}

The etiology and pathogenesis of infertility due to ovulation disturbance is thought as the kidney weakness, liver depression, internal retention of phlegm dampness, blood stasis by TCM. TCM thinks that the eggs are difficult to mature if the kidney is weak, which is the basic reason of ovulation dysfunction. Insufficiency of the kidney Yang can cause the lake of motivation in ovulation. If the liver is depressed, the eggs are difficult to drain. Internal retention of phlegm dampness and blood stasis can also cause the drainage of eggs.

WM thinks that normal ovulation cycle is based on the whole regulation function of hypothalamus-pituitary-ovary axis and normal secretory function of oa- 
rium. If any link of the axis is abnormal, the factors causing infertility can occur such as follicular agenesis, follicular atresia, anovulation, luteal insufficiency etc.

Ovulation is a complicated physiological process. The obvious feature of the female reproductive system is cyclic change, which is controlled by the adjusting among hypothalamus, hypophysis and oarium. Certainly, the female reproductive system is affected by the micromodulation of nerve center and other endocrine glands. Ovulation is a process that egg cell escapes from ovarian follicle. It is the key aspect of the whole reproductive process. If the function of any link of the above adjusting process is imbalance, ovulation dysfunction infertility can occur [11] [12] [13].

As for the treatment, TCM chooses the drugs of tonifying the liver and kidney, dispelling phlegm and eliminating dampness, activating blood and dissolving stasis. Potion is often combined with acupuncture and physiotherapy. WM generally adopts the drugs of clomiphene or chorionic gonadotropin, sometimes combined with surgery. Treatment of WM has the characteristics of high ovulation induction rate and low pregnancy rate. Sometimes side effects can occur. The study and practice show that the combination of TCM and WM is better in increasing the ovulation induction rate and pregnancy rate meanwhile decreasing the side effects than TCM or WM only [14]. The difference is obvious $(\mathrm{P}<$ 0.05) (Figure 1).

\subsection{Infertility Due to Salpingitis}

Salpingitis can induce infertility. Salpingitis is caused by the germs such as diplococcus gonorrhoeae, mycobacterium tuberculosis, chlamydia trachomatis etc. By the pathological change due to the infection difference of channels of transmission, different inflammatory syndrome can be observed such as oviduct mucositis, oviduct empyema, tubal interstitial inflammation, perisalpinitis etc. Long term inflammatory stimulation can cause oviduct fimbria atresia, destruction of tubal mucosa, which leads the injury of function and structure of fallopian tube due to complete obstruction of fallopian tube, hydrosalpinx. TCM thinks that

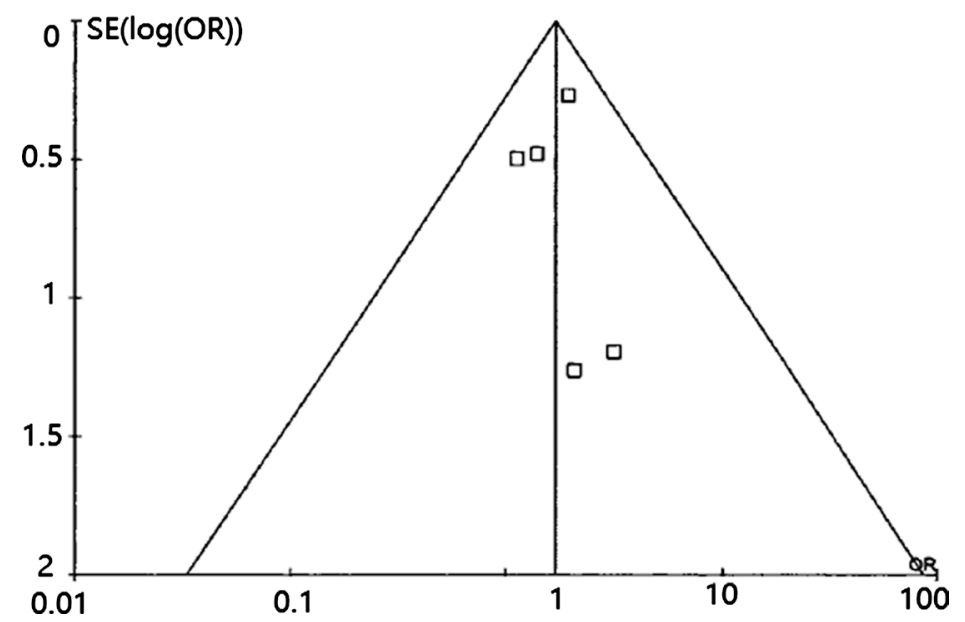

Figure 1. Funnel plot of comparison: TCM + WM vs WM (Figure 12 in literature [13]). 
acute salpingitis is induced by the flourishing of toxic heat and chronic salpingitis is caused by uncurable acute salpingitis. WM thinks that salpingitis is because of the involvement of multiple cytokines such as interleukin, pelvic inflammatory disease, infectious diseases [15], endometriosis [16] or some surgical reasons such as myositis uteri, adnexitis and pelvic infection due to abortion.

TCM often adopts the methods of detoxification, turbidity elimination, deobstruent and acupuncture for treatment [17] [18]. The common treatment methods of WM are microwave hyperthermia, hydrotubation, stem cell therapy and the combination of these methods. Microwave hyperthermia has the effects of anti-inflammatory, detumescence and reducing exudation by using the heat effects of microwave. Hydrotubation is often combined with microwave hyperthermia or TCM [19], stem cell therapy cures chronic salpingitis by stem cell transplantation [20].

Clinically, TCM is often combined with WM to treat infertility due to salpingitis. For example, patients are given oral Chinese medicine, herbal retention enema and iontophoresis or tubal hydrotubation combined with Chinese herbs before/after hysteroscopic laparoscopic surgery [21] [22] [23] [24].

\subsection{Infertility Due to Endometrial Dysreceptivity}

Endometrial receptivity means the receptivity of maternal endometrium toblastocyst. Endometrial dysreceptivity is one of the main pathogenies of female infertility. Clinical practice shows that TCM for tonifying kidney combined with WM has perfect effects on improving of endometrial receptivity due to kidney deficiency type endometrial dysreceptivity [25] [26]. For patients of infertility with luteal insufficiency, TCM combined with progesterone is a good method of treatment [27] [28]. Some researchers find that WM such as estrogen or clomiphene combined with TCM such as invigorate kidney and activate blood circulation prescription or Cangfu Daotan decoction has perfect effects on improve the endometrial blood flow and receptivity [29] [30] [31] [32] [33] and is better than that by only WM.

Liang's [34] study shows that estradiol valerate combined with nourishing kidney and blood decoction can obviously improve the clinical symptom such as soreness and weakness of the lumbar region, fatigue, lacking in strength etc. It can also change the endometrial thickness and type and blood flow. Endometrial receptive lesions can be recovered greatly and the clinical pregnancy rate can increase.

\subsection{Infertility Due to Endocrine Dysfunctional}

The incidence rate of infertility increases recently with the quickening pace of life. The main clinical features include irregular menstruation, disorder of ovarian axis regulation. The study shows that the effective and safe treatment is TCM combined with WM. WM accompanying the decoction for promoting blood circulation and removing blood stasis has obvious effects [35] [36]. 
For example, different TCM recipes are adopted in different stages of the menstrual cycle to adjust the meridians and vasculature [37]. Based on the use of TCM, clomiphene or chorionic gonadotropin is used at the same time. In this way to make the menstruation and fertility recovered (Table 1 ).

\subsection{Infertility Due to Polycystic Ovarian Syndrome}

Polycystic ovarian syndrome (PCOS) is one of the endocrine disorder syndromes. The clinical characteristics are polycystic ovary, insulin resistant, rare ovulation or even anovulation et al. It is also one of the reasons for infertility.

To treat this disease, promoting blood circulation and regulating menstruation, soothing liver and relieving depression based on the use of clomiphene is the main method. The effects of this method are more obvious than that by only TCM or WM [38]. Hua [39] adopted the recipe invigorate kidney and activate blood circulation combined with trazole to treat patients of infertility due to kidney deficiency and blood stasis type PCOS. Chen et al. [40] adopted acupuncture and trazole and HCG to treat infertility due to obstinacy PCOS. Yi et al. [41] adopted electropuncture combined with regulating menstruation and expelling phlegm to treat infertility of PCOS patients by decorporation with letrozole [42]. These clinical practices show good effects.

\subsection{Infertility Due to Luteal Insufficiency}

Luteal insufficiency means that the luteal dysplasia and incomplete function after ovulation. Accordingly, the progesterone synthesis and secretion are insufficient or the reactivity of endometrium to progesterone decreases, which leads to the slow development of endometrium in secretory phase, and then hinder the implantation and development of fertilized eggs. This is one of the most important reasons of infertility.

WM uses hormone therapy, and there are certain limitations in the treatment of drug side effects. TCM uses dialectical treatment, but the treatment cycle is longer. The combination of TCM theory and WM has been accepted and respected by most scholars for the treatment of sterility.

The common methods of treatment include self-made prescription of TCM combined with WM, syndrome differentiation of TCM combined with WM, artificial cycle of TCM combined with WM, special prescription of TCM combined with WM [43].

Table 1. Comparison of two groups after treatment of 6 menstrual cycles [37].

\begin{tabular}{|c|c|c|c|c|}
\hline NO. group & Number of patients & Number of fertility & Number of infertility & Infertility rate \\
\hline Observation & 31 & 25 & 6 & 80.6 \\
\hline Comparison & 31 & 15 & 16 & 48.4 \\
\hline$x^{2}$ & & & & 7.05 \\
\hline $\mathrm{P}$ & & & & 0.008 \\
\hline
\end{tabular}




\subsection{Infertility Due to Lhuteinized Unruptured Foilicle Syndrome}

Lhuteinized Unruptured Foilicle Syndrome (LUFS) is that the ovarian follicle matures but does not fracture. Therefore, patients can not normally shed ovulation two days after the peak of luteinizing hormone, which directly affect the infertility. For this type of disease, treatment by using human chorionic gonadotropin + nourishing kidney, activating blood circulation and promoting excretion decoction combined with has obvious effects [44].

\subsection{Infertility Due to Pelvic Infection and Endometrial Polyps}

Pelvic infection can cause the tubal injury, adhesion and chronic salpingitis. Accordingly, the function of transporting eggs and fertilizing eggs will decrease, so the risk of infertility and ectopic pregnancy will increase. For this type of infertility, on one hand, pelvic block injection of anti-infective drugs is chosen to restrain the release of key inflammatory mediators, on the other hand, intermediate frequency pulse therapy and electrode heating method are used to increase the local temperature and improve local blood circle [45]. Meanwhile, plaster with effects of promoting Qi and Tongluo is often used as adjuvant therapy.

\subsection{Infertility Due to Endometrial Polyps}

Endometrial polyps are the localized hyperplasia of stratum basal layer of endometrial and consist of endometrial gland and stroma. It is the common reason of infertility and abnormal uterine bleeding. Hysteroscopy surgery is the main method of WM to treat endometrial polyps. However, some complications often occur after surgery, such as hyperhydration syndrome, or even acute left heart failure, brain edema etc. TCM treats endometrial polyps with ovulation promoting decoction, methods of promoting blood circulation and removing blood stasis or invigorating spleen, tonifying kidney and resolving phlegm, whose side effects are small [46].

\section{Conclusions}

Infertility, one of the difficult complicated diseases, becomes more and more in recent years with the quickening pace of life and the increase of work pressure. Researchers and doctors have presented many theories and therapeutic methods on this type disease. This paper summarizes the treatment of female infertility by integrated TCM and WM. The explanations of pathology and therapeutic method are discussed. The imbalance or lesson of any link of the reproductive system can cause infertility. The treatment plan is determined according to which link is in disorder or sickness to induce infertility. It is shown that the treatment of by integrated TCM and WM can not only improve the ovulation rate and pregnancy rate, but also decrease the complications. The effects are better than that by TCM or WM only.

In the future study, experiments should be fully valued, and new clinical practices should be processed more and more. By using modern science and tech- 
nology, better methods of treatment should be built.

\section{Conflicts of Interest}

The authors declare no conflicts of interest regarding the publication of this paper.

\section{References}

[1] Wang, C.H. (2015) Progress in the Study of Anovulatory Infertility. Thesis for Master Degree, Beijing University of Chinese Medicine, Beijing. (In Chinese)

[2] Wang, A.T., Mulian, R.J., Lane, M.A., et al. (2012) Treatment of Hyperprolactinemia: A Systematic Review and Meta-Analysis. Systematic Reviews, 1, 33. https://doi.org/10.1186/2046-4053-1-33

[3] Cao, Y.Y., Yu, Y., Zou, H., et al. (2018) Research Progress of Hyperprolactinemia Infertility in the Study of Traditional Chinese Medicine and WM. Journal of Changchun University of Chinese Medicine, 34, 599-602. (In Chinese)

[4] He, J.H. and Lin, H.M. (2017) Professor Lin Hanmei's Experience in Treating Hyperprolactinemia with Traditional Chinese Medicine. Popular Science and Technology, 19, 44-46. (In Chinese)

[5] Lin, Q.Y. (2016) Study on the Progress of Traditional Chinese and WM on Diminished Ovarian Reserve and the Discussion on the Law of TCM. Thesis for Master Degree, Beijing University of Chinese Medicine, Beijing. (In Chinese)

[6] An, L.F. (2015) The Correlation Research on Tfh, IL-21 and Other Factors with Producing Autoantibodies in Immune Infertility. Lanzhou University, Lanzhou.

[7] Poppe, K., Glinoer, D., Tournaye, H., et al. (2006) Thyroid Autoimmunity and Female Infertility. Verhandelingen-Koninklijke Academie voor Geneeskunde van Belgie, 68, 357-377.

[8] Liu, R.F. and Wang, M.H. (2003) Pathogenesis and Treatment of Infertility Due to Female Immunity. Fujian Journal of Traditional Chinese Medicine, 34, 26-27. (In Chinese)

[9] Yu, T.G. (2000) Acquaintance of Treatment of Infertility Due to Female Immunity. Practical New Medicine, 2, 1119-1120. (In Chinese)

[10] Hu, W.Y. and Long, T. (2020) Progress on the Immune Infertility Treatment of Chinese and Western Medicine. China's Naturopathy, 28, 112-115. (In Chinese)

[11] Chen, S.J. (2006) Clinical Study on the Treatment of Anovulatory Infertility. International Medicine and Health Guidance News, 12, 34-36. (In Chinese)

[12] Wang, N.N. (2010) Study Progress of Ovulation Dysfunction Infertility. Thesis for Master Degree, Beijing University of Chinese Medicine, Beijing. (In Chinese)

[13] Huang, P.F. (2010) Evaluation on the Efficacy and the Rules of Syndrome Differentiation and Treatment in Chinese Medicine of Ovulatory Disorder Infertility. Thesis for PhD, Guangzhou University of Chinese Medicine, Guangzhou. (In Chinese)

[14] Zhao, R.H. (1994) Experience of Xu Runsan on the Infertility by Treatment from Liver and Kidney. Chinese Archives of traditional Chinese Medicine, 2, 20-21. (In Chinese)

[15] Han, L. and Sun, L. (2012) Analysis of 132 Cases of Tubal Inflammatory Obstructive Infertility Treated by Traditional Chinese. Chinese Journal of Experimental Traditional Medical Formulae, 18, 258-259. (In Chinese)

[16] Rachel, A.L., Ovrang, D., Ertans, S., et al. (2002) Peritoneal Fluid, Endometriosis, 
and Ciliary Beat Frequency in the Human Fallopian Tube. The Lancet, 360, 1221-1222. https://doi.org/10.1016/S0140-6736(02)11247-5

[17] Liu, J.J. and Zhang, W.H. (2016) Zhang Wenhong's Experiences in the Treatment of Oviduct Obstructive Infertility. World Journal of Integrated Traditional and Western Medicine, 11, 1351-1352. (In Chinese)

[18] Yu, Y.H. (2017) Clinical Analysis of Acupoints Commonly Used in the Therapy of Tubal Inflammatory Infertility by Acupuncture and Moxibustion. Electronic Journal of Clinical Medical Literature, 4, 7939-7942. (In Chinese)

[19] Qu, X.F. (2011) Efficacy Analysis of Hydrotubation Liquid Intraoperative Adding Comprehensive Treatment for Oviduct Infertility of Tubal. Medical Information, 24, 2987-2988. (In Chinese)

[20] Li, Z., Zhang, Z., Chen, X., et al. (2017) Treatment Evaluation of Wharton's Jelly-Derived Mesenchymal Stem Cells Using a Chronic Salpingitis Model: An Animal Experiment. Stem Cell Research \& Therapy, 8, 232. https://doi.org/10.1186/s13287-017-0685-0

[21] Zhong, H.H., Ma, X. and Shen, G.Z. (2018) Clinical Analysis of Traditional Chinese Medicine Combined with Hysteroscopy and Laparoscopy in the Treatment of Tubal Obstruction Infertility. Chinese Remedies \& Clinics, 18, 573-575. (In Chinese)

[22] Wu, T.R. (2014) Clinical Experience of 40 Cases of Tubal Inflammatory Obstructive Infertility Treated by Integrated Traditional Chinese and Western Medicine. Journal of Guiyang College of Traditional Chinese Medicine, 36, 91-92. (In Chinese)

[23] Obstetrics and Gynecology Branch of Chinese Medical Association (2007) Guidelines for Clinical Diagnosis and Treatment-Obstetrics and Gynecology. People's Health Press, Beijing, 22-24. (In Chinese)

[24] Hao, C.L. and Chen, P. (2019) Research Progress of Tubal Inflammatory Infertility. Guangming Journal of Chinese Medicine, 34, 1611-1614. (In Chinese)

[25] Zhu, Y.M., Feng, Y. and Ning, Y. (2017) Meta Analysis of Traditional Chinese Medicine in Improving the Endometrium of Patients with Renal Infertility. Lishizhen Medicine and Materia Medica Research, 28, 1273-1276. (In Chinese)

[26] Hu, T.H., Gu, W.H. and Du, M. (2015) Clinical Research of Ankun Zhongzi Pill on Endometrial Receptivity of Infertility Induced by Luteal Phase Deficiency. China Journal of Traditional Chinese Medicine and Pharmacy, 30, 923-925. (In Chinese)

[27] Zhang, S.S. (2018) Effect of Bushen Decoction Combined with Progesterone on Endometrial Receptivity of Infertility with Luteal Insufficiency. Journal of Practical Traditional Chinese Medicine, 34, 244-245. (In Chinese)

[28] Li, Y.L. (2017) Effect of Ankun Decoction on Endometrial Receptivity of Infertility Patients with Luteal Insufficiency. Asia-Pacific Traditional Medicine, 13, 140-141. (In Chinese)

[29] Li, Q. (2017) Effect of the Modified Yangmo Decoction Combined with Estrogen on Endometrial Receptivity and Uterine Hemodynamics in Patients with Kidney Deficiency and Blood Stasis Type Thin Endometrium. Journal of Bengbu Medical College, 42, 1517-1521. (In Chinese)

[30] Feng, X.M. (2016) Bushen Huoxue Decoction Combined with Estradiol Valerate on Pregnancy Outcome and Endometrial Receptivity of Patients with Infertility. Shanxi Journal of Traditional Chinese Medicine, 37, 1504-1506. (In Chinese)

[31] Li, X.Y., Yang, Q. and Wang, Q. (2017) Effects of Cangfu Daotan Decoction Combined with Clomiphene on Serum Hormone Level and Endometrial Receptivity in Patients with Polycystic Ovary Syndrome Combined with Infertility. China Phar- 
macy, 28, 3698-3701. (In Chinese)

[32] Wang, Y.F. (2016) Preliminary Assessment on Treating Ovulatory Disorder Infertility by the Bushen Therapy. Clinical Journal of Chinese Medicine, 8, 61-62. (In Chinese)

[33] Yang, D.X., Hou, L. and Li, H.M. (2019) Advances in Study of Endometrial Tolerance in Patients with Infertility Caused by Chinese and Western Medicine. Liaoning Journal of Traditional Chinese Medicine, 46, 2006-2009. (In Chinese)

[34] Liang, D. (2019) Clinical Study on the Effect of Integrated Traditional Chinese and Western Medicine on Endometrial Receptivity in Patients before FET. Thesis for Master Degree, Yunnan University of Chinese Medicine, Kunming. (In Chinese)

[35] Tan, X.Y. (2019) Effect of Integrated Traditional Chinese and Western Medicine on Endocrine Dysfunctional Infertility. Contemporary Medical Symposium, 17, 198-199. (In Chinese)

[36] Yan, L. (2019) Clinical Observation of Integrated Traditional Chinese and Western Medicine in Treating Infertility. Chinese Naturopathy, 27, 57-58. (In Chinese)

[37] Li, T.T. and Zhao, Z.H. (2020) Clinical Efficacy of Integrated Traditional Chinese and Western Medicine in the Treatment of Infertility Due to Polycystic Ovary Syndrome. Journal of Mathematical Medicine, 33, 94-95. (In Chinese)

[38] Cui, L.Y., Cui, R. and Su, Y. (2019) Clinical Observation on Infertility Caused by Polycystic Ovary Syndrome Treated by Integrated Traditional Chinese and Western Medicine. China Practical Medical, 14, 121-123. (In Chinese)

[39] Hua, C.F. (2018) Clinical Observation of Bushen Huoxue Decoction in Promoting Ovulation Cycle of Polycystic Ovary Syndrome. Nanjing University of Chinese Medicine, Nanjing. (In Chinese)

[40] Chen, Y.T., Li, Y.M., Luo, J.L., et al. (2017) Clinical Observation of Tong Yuan Needling Method plus Letrozol and HCG for Refractory Sterility Caused by Polycystic Ovary Syndrome. Shanghai Journal of Acupuncture and Moxibustion, 36, 692-696. (In Chinese)

[41] Yi, Y., Zhang, Y.C., Zhang, H., et al. (2018) Clinical Therapeutic Effects of Acupuncture Combined with Chinese Herbal Medicine on Infertility of Polycystic Ovary Syndrome in the Patients with Ovulation Induction with Letrozole. Chinese Acupuncture \& Moxibustion, 38, 27-32. (In Chinese)

[42] Rao, L. and Zhu, H.L. (2019) Progress of Chinese and Western Treatment of Infertility Caused by Cystic Ovarian Syndrome. Hubei Journal of TCM, 41, 60-62. (In Chinese)

[43] Wang, M.M. and Liu, J.X. (2019) Research Progress on Combined Chinese and Western Medicine Treatment of Luteal Dysfunction Infertility. World Latest Medicine Information (Electronic Version), 19, 63-65. (In Chinese)

[44] Cao, X.M. and Gu, P. (2019) Clinical Study on Treatment of Infertility Caused by Luteinized Unruptured Folicle Syndrome with Combination of TCM and Western Medicine. Journal of Practical of Traditional Chinese Medicine, 35, 1210-1211. (In Chinese)

[45] Zheng, R.J., Cui, L., Ma, C.F., et al. (2019) Treatment of 60 Cases of Tubal Inflammatory Infertility with Integrated Chinese and Western Medicine. TCM Research, 32, 13-16. (In Chinese)

[46] Gao, D. and Han, L. (2018) Progress of Chinese and Western Study on the Endometrial Polyps and Infertility. Xinjiang Journal of Traditional Chinese Medicine, 36, 102-105. (In Chinese) 